grief support team following stillbirth. Am J Obstet Gynecol 1983;146(8):877-881.

9. Condon JT. Prevention of emotional disability following stillbirth: The role of the obstetric team. Aust NZ J Obstet Gynaecol 1987;27:323-329.

10. Davis DL, Stewart M, Harmon RJ. Perinatal loss: Providing emotional support for bereaved parents. Birth 1988; 15(4):242-246.

11. Fetus and Newborn Committee, Canadian Pediatric Society. Support for parents experiencing perinatal loss. Can Med Assoc J 1983;129:335-339.

12. Hildebrand WL, Schreiner RL. Helping parents cope with perinatal death. Am Fam Physician 1980;22(5):121-125.

13. Kennell JH, Slyter $\mathrm{H}$, Klaus $\mathrm{MH}$. The mourning response to the death of a newborn infant. N Engl J Med 1970;283(7): 344-349.

14. Hughes $P$. The management of bereaved mothers: What is best? Midwives Chron 1987;100(1195):226-229.
15. Tadmor CS. A crisis intervention model for a population of mothers who encounter neonatal death. J Primary Prevention 1986;7(1):17-26.

16. Rowe J, Clyman R, Green C, Mikkelson C, Haight J, Ataide $\mathrm{L}$. Follow-up of families who experience a perinatal death. Pediatrics 1978;62(2):166-170.

17. Lamb JM Sr. How to Start a Support Group. St. Joseph's Health Center, MO: SHARE.

18. Ilse $S$, Blackburn C. Development of a comprehensive follow-up care plan after perinatal and neonatal loss. J Perinat Neonat Nurs 1988;2(2):23-33.

19. Malone B, McElwain C. Role of the nurse during a pregnancy loss. In: Woods \& Esposito, Pregnancy Loss: Medical Therapeutics and Practical Considerations. Cincinnati: Williams \& Wilkins, 1987:120-131.

20. Woods J. Stillbirth, In: Woods \& Esposito, Pregnancy Loss: Medical Therapeutics and Practical Considerations. Cincinnati: Williams \& Wilkins, 1987:51-74.

\title{
Commentary: Providing Versus Packaging Support for Bereaved Parents After Perinatal Loss
}

\author{
Irving G. Leon, Ph.D.
}

It is no easy task to help parents cope with the death of a baby or pregnancy loss. In addition to confronting the often intense emotions of parents who may be traumatized by such a sudden death, medical caregivers must simultaneously manage their own particular blend of grief, including sorrow, bitterness, and helplessness. They can feel bombarded from outside as well as from within. Brown implicitly recognizes a fact that is often overlooked in this area.

For both personal and professional reasons, some caregivers are extremely sensitive to the needs of bereaved parents and are enormously helpful. Many others may experience this death as so distressing that they tend to shy away from empathizing with parental grief, either by overtly denying the reality of the loss (the way of the past) or routinely arming themselves with the "right" words or procedures (the way of the present). In either instance, emotionally engaging with the bereaved is avoided. By developing the kind of volunteer nursecounselor program for providing support that Brown outlines, there is a good chance that those who specialize in this area will have the motivation,

Irving Leon is Lecturer in the Departments of Psychiatry and Psychology at The University of Michigan, Ann Arbor, MI, and author of When a Baby Dies: Psychotherapy for Pregnancy and Newborn Loss. stamina, and sensitivity to view each loss as the highly individual event that it is.

We have to make greater use of volunteers. Although Brown cites the need for professional confidentiality in excluding nonmedical counselors from the team, I suspect nobody is as effective as another bereaved parent to comfort, understand, and normalize the reactions of parents who have just suffered such a loss. This will be especially true for counselors who can appreciate the wide range of responses involved. Although mutual support groups such as Source of Help in Airing and Resolving Experiences (SHARE) are invaluable, it should be remembered that only a small proportion of bereaved parents ever attend them. Lay volunteer counselors should be more fully integrated into the hospital support system as a basic component of care for all bereaved parents. In these fiscally troubled times, neglecting such a cost-effective resource is an absolute shame.

Brown is to be applauded for presenting specific cases to illustrate the wide range and complexity of clinical examples. In the first case, what may have seemed to the physician like an obvious need to end the pregnancy, due to the discovery of serious fetal anomaly plus the mother's health in jeopardy, was complicated by a history of perinatal loss and infertility. This creates uncertainty as to whether the woman would ever have any successful births, and makes the decision to terminate this pregnancy es- 
pecially difficult and painful. In the second case, it was not surprising to encounter the tug-of-war between the adolescent pregnant woman and her parents over controlling arrangements, illustrating the same conflicts over separation-individuation within this family that may have contributed to this young woman becoming pregnant.

Too many papers are long on detailed recommendations and short on actual clinical examples. They leave the reader with the uneasy feeling that there is one correct way of approaching all families (despite the usual disclaimers). A comment by Davis and colleagues (1) is especially relevant: "It is also important to realize that every parent is different, and a statement that comforts one may offend another" (p. 244). I would have liked to see even more detailed accounts of interactions. Klaus and Kennell's (2) presentation of transcripts of parent-caregiver interchange continues to be a worthy model to emulate for increasing communication among professionals about how these situations are handled and improving clinical skills.

It is worth highlighting Brown's central point: "During initial and subsequent visits, all interventions are guided by one key concept: the meaning and significance of the loss to the mother, her partner, and her family." She succinctly indicates that not only is the loss experienced individually, but three separate dimensions must be consideredindividual, marital, and family. Other than a cursory mention in her first sentence, she does not, however, give sufficient attention to the many ways in which these losses are experienced. This is unfortunately representative of the increasing tendency in this area to consider perinatal loss as solely the loss of a baby, thereby minimizing the other important ways in which the event may become an assault on self-esteem, interference with one's developmental aspirations to parent, and catalyst to reviving earlier unresolved losses or conflicts (3).

Understanding these diverse repercussions does not in itself demand a mental health background. However, a psychotherapist schooled in several orientations (particularly psychoanalytic and family) would be a valuable member of a multidisciplinary support team. Such a therapist is ideally suited to appreciate the complicated dynamics in many of these cases, facilitating the development of optimal interventions. Although short-term psychotherapy has a place in helping some bereaved parents and families (4), the temptation by a mental health professional to "pathologize" the wide range of normative reactions or routinely recommend therapy has to be resisted. Sadly, the anachronistic and profoundly destructive view that pregnancy loss is motivated by unconscious conflicts over motherhood that may be resolved through psychoanalysis (5) evidently persists among some psychoanalysts and must be dispelled. This has not prevented other psychoanalysts from providing some of the most sensitive and insightful understandings of the impact (not causes) of this tragedy, however $(6,7)$.

It is necessary to apply broad guidelines and checklists to ensure standards and continuity of care among all health professionals who may not always be able to communicate directly with each other. But it now appears that the tail is wagging the dog. It is disheartening to observe the proliferation of detailed checklists and behavioral protocols listing dozens of instructions (8-10). Despite the wellmeaning intent of their creators and the sensitivity of individual suggestions, such protocols can lead to a regimented, assembly-line approach to these losses. This impedes attempts to attune to parents individually and empathically - the very essence of providing support $(11,12)$.

For example, the now standard policy of encouraging parents to view and hold their dead child, in Furman's words, "is often pursued as an end in itself rather than as a part of an empathic professional relationship" (4). It was gratifying to observe that in two of the four cases in Brown's report, when the parents declined to view their children, this was not assumed to portend unresolved grief. The clinical wisdom of gently encouraging such contact by observing how it is valuable for many parents is not being challenged. However, the belief that such contact inevitably or exclusively ensures resolution of these losses is not supported by quantitative studies (3). It also confuses the internal process of mourning (i.e., detaching oneself from the relationship with the deceased) with behavioral interactions that, while facilitating mourning, are not indispensable to it. In both of Brown's cases in which contact was declined, the counselor effectively empowered the parents to do what was right for themselves (as opposed to following a set protocol), thereby reducing the traumatizing helplessness often resulting from these losses.

Brown should use caution with respect to her proposed "how-to" book for case management. General guidelines are valuable as long as they do not become so overly narrow and specific that, instead of facilitating caregiver sensitivity to individualized nuances, they become an obstacle to emotionally engaging parents. With that in mind, I was disappointed that Brown failed to include SwansonKauffman's (13) very useful description of the in- 
gredients of optimal nursing care after pregnancy loss. Those facets of care mirror, in fact, the crucial therapeutic factors in my own psychotherapy with bereaved mothers (4). Ultimately, it will be a caregiver's ability to listen, understand, and respond empathically to the family's experience of this loss that will help the most.

\section{References}

1. Davis DL, Stewart M, Harmon RJ. Perinatal loss: Providing emotional support for bereaved parents. Birth 1988; 15(4):242-246.

2. Klaus MH, Kennell JH. Parent-infant bonding. St. Louis: CV Mosby, 1982.

3. Leon I. The psychoanalytic conceptualization of perinatal loss: A multi-dimensional model. Am I Psychiatry in press.

4. Leon I. When a Baby Dies: Psychotherapy for Pregnancy and Newborn Loss. New Haven: Yale University Press, 1990.
5. Pines D. Pregnancy, miscarriage and abortion. A psychoanalytic perspective. Int $J$ Psychoanal 1990;71:301-307.

6. Furman E. The death of the newborn: Care of the parents. Birth Fam J 1978;5(4):214-218.

7. Lewis E, Bourne S. Perinatal death. Baillieres Clin Obstet Gynaecol 1989;3:935-953.

8. Ryan PF, Côté-Arsenault D, Sugarman LL. Facilitating care after perinatal loss-A comprehensive checklist. JOGN Nurs 1991;20:385-389.

9. Carr D, Knupp S. Grief and perinatal loss. JOGN Nurs 1985;14:130-139.

10. Hutti MH. A quick reference table of interventions to assist families to cope with pregnancy loss or neonatal death. Birth 1988;15(1):33-35.

11. Leon I. Perinatal loss: Choreographing grief on the obstetric unit. Am J Orthopsychiatry 1992;62:7-8.

12. Leon I. Perinatal loss: A critique of current hospital practices. Clin Pediatr in press.

13. Swanson-Kauffman K. Caring in the instance of unexpected early pregnancy loss. Topics Clin Nurs 1986;8:3746. 